\title{
Helically Localized Ballooning Instabilities in Three-Dimensional Tokamak Pedestals
}

\author{
T. B. Cote, C. C. Hegna \\ Department of Engineering Physics, University of Wisconsin-Madison, Madison, Wisconsin, 53706 USA \\ M. Willensdorfer, E. Strumberger, W. Suttrop, H. Zohm, ASDEX Upgrade Team \\ Max-Planck-Institute for Plasma Physics, 85748 Garching, Germany
}

\begin{abstract}
Recent experimental observations have found toroidally localized MHD instabilities in the plasma edge during operation with applied magnetic perturbations on ASDEX Upgrade in H-mode with low collisionality $\left(v^{\star} \approx 0.4\right)$. Large edge plasma displacements are induced by a stable kink response to the 3D magnetic perturbations. This kink response results in localized changes of geometric quantities, which in turn leads to the localization of MHD instabilities in the plasma edge. Infinite-n ideal MHD ballooning theory is shown to predict the existence of these instabilities, as well as the observed toroidal localization. Utilizing 3D VMEC equilibria, the local geometric parameters determining ideal stability, include the local magnetic shear, normal curvature, and geodesic curvature, are calculated for experimentally relevant conditions. It is found that these shaping parameters have significant levels of $3 \mathrm{D}$ variation, with the local magnetic shear being the dominant factor behind changes in the local geometry. This behavior leads to a significant decrease in the stabilizing line-bending energy for certain field lines, resulting in the localization of the ballooning instability. Furthermore, it is observed that a finite amount of magnetic perturbation (and subsequent edge perturbation) is necessary to modify the local geometry and excite the localized instability, leading to a threshold behavior.
\end{abstract}




\section{Introduction}

Mitigation or complete suppression of edge localized modes (ELMs) is critical to the success of future fusion devices in order to avoid potentially harmful transient heat loads in tokamak $\mathrm{H}$-mode operation. Application of non-axisymmetric magnetic perturbations (MPs) is a prominent method to achieve ELM mitigation [1] or suppression [2] [3]. However, the physical mechanism behind this approach is not yet fully understood. Three-dimensional magnetic perturbations have previously been shown to impact edge transport and turbulence during ELM suppression [4, 5], which may play a key role in the suppression process. Studies at ASDEX Upgrade [6, 7, 8], MAST [6], and DIII-D [9] have shown that ELM suppression and mitigation are optimized when the poloidal mode spectrum of the externally applied MPs excite stable kink modes at the edge which are strongly amplified by the plasma response [10]. This kink response has been shown to change the local 3D geometry of the plasma, creating 3D toroidally localized ideal MHD ballooning instabilities in the tokamak pedestal [11]. In this work, we look to understand these ballooning instabilities and the underlying mechanism of the 3D localization.

The stable kink response causes 3D displacement of the plasma boundary and associated 3D deformations of the flux surfaces in the pedestal region. Non-axisymmetric boundary deformations are important to understand as changes in 3D geometry can play a key role in edge turbulent transport [12] and ELM stability [13, 14]. These 3D boundary displacements have been studied in multiple machines and reviewed in Ref. [15], which concludes that the magnitude of the edge displacement scales linearly with the applied MPs calculated by vacuum field modeling. However, in some cases, it was observed that vacuum modeling drastically underestimated the 3D displacement due to the stable kink response [16] [17]. In recent experiments at ASDEX-Upgrade, the stable ideal kink mode was found to dominate the edge plasma response for ELM mitigated discharges with low collisionality $\left(v^{*}<0.4\right)[18,19]$. In these experiments, the observed displacement was nearly four times larger than the predicted vacuum field when the applied poloidal mode spectrum was optimized for the kink response [19].

Additionally, these ASDEX-Upgrade experiments have found sufficiently large 3D magnetic perturbations can lead to destabilization of MHD modes in the plasma edge region [11]. Infinite- $n$ ballooning theory [20] is used to estimate the impact of the MPs on these instabilities for numerous 3D MHD configurations [21, 12, 22]. Theoretical studies have shown that modulation of the local magnetic shear due to external MPs changing the magnetic geometry of the plasma edge can significantly impact local ballooning stability [12]. In this work, we utilize a ballooning model to study the effects of the MP driven 3D kink response on ballooning stability of ASDEX-Upgrade equilibria. We assert that changes of the local magnetic geometry due to 3D magnetic perturbations and large edge displacement are the dominant mechanism for the changes and localization of MHD activity observed in the ASDEX-Upgrade experiments.

In the following section, the relevant ASDEX-Upgrade experiments are described. Section 3 covers the three-dimensional equilibria constructed using VMEC, which are representative of the ASDEX-Upgrade experiments. In section 4, the infinite-n ballooning model is used to evaluate the stability of the experimentally relevant equilibria in the presence of applied 3D magnetic perturbations. It is found that ideal ballooning theory is sufficient to predict the localized increase in MHD activity observed in the experiment. Furthermore, we analyze and quantify the effects of various factors on the behavior of the instability, including the strength of the MPs, pressure, and the local magnetic geometry. In particular, the 3D structure of the magnetic curvature and local 
magnetic shear are shown to impact the local stability of the system. While prior studies noted the prevalence of 3D responses near rational surfaces due to Pfirsch-Schlüter responses [12], we find significant distortions of the geometric quantities in the pedestal region despite the absence of a dominant rational surface. In section 5, these results are summarized. Additionally, the discussion is broadened to include the potential impact of these results on a range of applications of 3D magnetic perturbation.

\section{Experimental background}

The stability calculations presented here are based on a series of ASDEX Upgrade experiments to measure the 3D displacement of the plasma boundary due to the stable kink modes excited by 3D magnetic perturbations [11, 19, 18]. The experiments had a toroidal field $\left|B_{T}\right|=$ of $2.5 \mathrm{~T}$, plasma current $I_{P}=800 \mathrm{kA}$, edge safety factor $q_{95} \approx 5.2$, and a core electron density $n_{e}=4.3$ $10^{-19} \mathrm{~m}^{-3}$. For full details on the setup for the ASDEX Upgrade for these discharges, we refer to [18, 19, 23]. The experimental series consists of six discharges with consistent global plasma parameters, which are summarized in Table 1 of [19].

External $\mathrm{n}=2$ MP-fields are applied under slow $(2-3 \mathrm{~Hz})$ rigid rotation. The amplitude of the 3D perturbations is set by the differential phase angle $\left(\Delta \varphi_{U L}\right)$ with peak plasma displacements of $\sim 11 \mathrm{~mm}$ for $\Delta \varphi_{U L} \approx-90^{\circ}$ for the highest $\beta_{N}$ equilibrium. The $\Delta \varphi_{U L} \approx-90^{\circ}$ configuration is optimal for exciting the stable edge kink modes experimentally [19].

During the experiments, an instability occurring at selected toroidal phases was observed [11]. Figure 1 shows electron cyclotron emission (ECE) time traces of perturbed electron temperature, taken near the top of the pedestal. The mode occurs near the zero crossing in the radial plasma displacement $\left(\xi_{n}=0\right)$, but only when going from positive to negative (i.e. at $t \approx 5.15,5.47,5.80$ in Fig. 1). Notably, localized MHD activity is absent when the plasma displacement has a zero crossing when going from negative to positive values. The instability is observed between ELM crashes with a large rotational frequency $(\sim 1 \mathrm{kHz})$ relative to the ELM frequency $(100 \mathrm{~Hz})$ and rotation frequency of the external MP field $(3 \mathrm{~Hz})$. For these experiments, the determined toroidal mode number for the MHD instability is in the range of $n=6-12$ [23]. Additionally, the instability appears strongest on the low-field side (LFS), and rotates toroidally opposite the MP-field and poloidally in the electron diamagnetic direction. The instability has also been observed in magnetic signatures on the LFS and in the Helium beam diagnostic [23].

\section{VMEC equilibria}

Three sets of 2D CLISTE [24, 25] equilibrium reconstructions are generated based on the experimental discharges [18, 19]. The reconstructions have slightly varied q-profiles and pressure profiles to account for small variations between discharges. Figure 2 shows the pressure profile and q-profile for the three cases, which have $\beta_{N}$ values of roughly 1.8 (red), 2.0 (blue), and 2.2 (green). All three of these equilibria have H-mode like pedestals, but different levels of bootstrap current which impacts the detailed shape of the q-profiles in the edge.

Using these 2D reconstructions as inputs, 3D equilibria were created with a free boundary version of VMEC [26, 27]. The equilibria each consist of 1000 flux surfaces, 17 toroidal mode numbers, and 26 poloidal mode numbers in order to assure sufficient resolution for the 3D 
calculations, which is particularly important for accurate calculation of the plasma displacement at the edge. The radial resolution is also critical for resolving the 3D geometric quantities studies here, as well as moderate convergence of the plasma currents at rational surfaces [28]. A total of 27 3D VMEC equilibria were generated from the three different input equilibria using nine values for the differential phase configurations $\left(\Delta \varphi_{U L}\right)$. In these cases, the variation of $\Delta \varphi_{U L}$ acts as a proxy for the magnitude of 3D displacement of the plasma boundary and is used for more direct comparison to the experimental observations [19]. Figure 3 shows the plasma displacement at the $q_{95}$ surface for the $\beta_{N} \approx 2.2, \Delta \varphi_{U L}=-93^{\circ}$ equilibrium. The largest displacements occur when $\Delta \varphi_{U L} \approx-90^{\circ}$ for both the experiment and VMEC equilibria.

The boundary displacement calculations for the 3D VMEC equilibria have been successfully tested against the experimental measurements, achieving a good agreement, even at the plasma boundary [19]. A full analysis of the 3D VMEC equilibria, including their relationship to experimental results and details specific to running VMEC for ASDEX Upgrade discharges are given in Ref. [19].

\section{$4 \quad$ Ideal ballooning analysis}

Ideal ballooning stability theory [20] is utilized to analyze the stability of the pedestal region under large 3D magnetic perturbations.

We first introduce the straight field line coordinate system $(\psi, \theta, \zeta)$ to describe the equilibrium magnetic field $\mathbf{B}=q(\psi) \nabla \psi \times \nabla \theta+\nabla \zeta \times \nabla \psi$ with safety factor profile $q=q(\psi)$. Here $\psi$ is the poloidal flux, $\zeta$ is the geometric toroidal angle, and $\theta$ is the poloidal angle that labels points along the field line. Normalizing with the major radius $\left(R_{0}\right)$ and the magnetic field on axis $\left(B_{0}\right)$, the lowest order incompressible ballooning equation is written

$$
\frac{1}{\sqrt{g}} \frac{\partial}{\partial \theta}\left[\frac{\left(1+\Lambda^{2}\right)}{|\nabla \psi|^{2}} \frac{1}{\sqrt{g}} \frac{\partial X}{\partial \theta}\right]+\hat{p} \frac{d \beta}{d \psi} \frac{\left(\kappa_{n}+\Lambda \kappa_{g}\right)}{|\nabla \psi|} X+\omega^{2} \frac{\left(1+\Lambda^{2}\right)}{|\nabla \psi|^{2}} X=0 .
$$

Here, $\omega$ is the ideal ballooning growth rate normalized by the Alfven frequency, $\hat{p}$ is a tuning parameter (see section 4.2), $\frac{d \beta}{d \psi}=\frac{2 \mu_{0}}{B_{0}^{2}} \frac{d p}{d \psi}$ is the normalized pressure gradient, $\kappa_{n}$ and $\kappa_{g}$ are the normal and geodesic curvatures, respectively, $\sqrt{g}=[\nabla \psi \times \nabla \theta \cdot \nabla \zeta]^{-1}$ is the Jacobian, and $\Lambda$ is the integrated local magnetic shear, given by

$$
\Lambda=\frac{|\nabla \psi|^{2}}{B}\left[q^{\prime} \theta_{k}+\frac{\nabla \psi \cdot \nabla \alpha}{|\nabla \psi|^{2}}\right]
$$

Here we introduce the normalized radial wave number of the mode $\theta_{k}$, as well as the helical angle, $\alpha=\alpha_{0}+\zeta-q \theta$, where $\alpha_{0}$ is an arbitrary constant. In this form, ideal ballooning theory reduces the properties of the $3 \mathrm{D}$ equilibrium to a series of ordinary differential equations along $\theta$ in a 3D phase space $\left(\alpha, \psi, \theta_{k}\right)$. For a given set of $\left(\alpha, \psi, \theta_{k}\right)$, the 3D VMEC equilibrium can be used to specify the necessary coefficients of the ballooning equation and subsequently used to solve the local eigenvalues for each field line.

The ideal MHD ballooning eigensolver used in this work is an extension of the STESA code [29, 30, 31, 32] and its successor developed for studying ballooning in local 3D equilibria [12, 22]. These codes are based on and utilize numerical techniques from the well studied ballooning solver 
COBRA [33], with STESA having been benchmarked directly against the COBRA solver [29]. Our extension of these codes utilizes the same underlying eigensolver, while allowing for more flexibility in chose of equilibria and additional tools for analysis of the equilibria and eigenvalue results. For this work, we utilize the 3D VMEC equilibria, which have been transformed into the necessary straight field line coordinates $(\theta, \zeta)$ utilizing the conversion factor $\lambda(\theta, \zeta)$ computed along side the VMEC equilibria [34], such that $\theta_{\text {vmec }}=\theta-\lambda(\theta, \zeta)$.

A summary of the full parameter space is given in Table 1. The procedure used starts with a scan over a range of $\psi$ and $\theta_{k}$ for the axisymmetric equilibria. This is used to determine the values $\psi_{0}$ and $\theta_{k, 0}$, which are the phase-space location of the most unstable field line for each of the three axisymmetric equilibria. The axisymmetric equilibria is then adjusted to be marginally stable using variation of the $\hat{p}$ value; the value for marginal stability is provided in Table 1. Each shot then uses a range of 9 values for the differential phase in the 3D-coils to vary the strength of the 3D magnetic perturbations, all of which are studied for $\alpha$ values ranging from 0 to $\pi$, since the systems have $N=2$ periodicity.

\begin{tabular}{|c|c|c|c|c|c|c|}
\hline Shot Nu. & $\sqrt{\psi_{0} / \psi_{a}}$ & $\theta_{k, 0}$ & $q\left(\psi_{0}\right)$ & $\beta_{N}$ & $\hat{p}$ & $\Delta \varphi_{U L}(\mathrm{deg})$ \\
\hline 33118 & 0.949 & $0.18 \pi$ & 5.36 & 1.8 & 1.21 & $-182,-132,-93-52,-2,49,88,128,178$ \\
33345 & 0.945 & $0.22 \pi$ & 5.06 & 2.2 & 1.04 & $-182,-132,-93-52,-2,49,88,128,178$ \\
33569 & 0.963 & $0.24 \pi$ & 5.51 & 2.0 & 1.12 & $-182,-132,-93-52,-2,49,88,128,178$ \\
\hline
\end{tabular}

Table 1: Overview of the complete parameter space used in this study.

\subsection{Localization of the ballooning instability}

Ballooning analysis show the ASDEX equilibria to have strong radial and toroidal localization of the ballooning instability. Figure 4 shows the ideal ballooning growth rates for 3D equilibrium of Shot 33345 with $\Delta \varphi_{U L}=-93^{\circ}$ throughout the $\left(\alpha, \psi, \theta_{k}\right)$ phase-space. The most unstable point is observed at $\sqrt{\psi / \psi_{a}}=0.945$ and $\theta_{k}=0.22 \pi$ for the $\alpha=0.51 \pi$ field line.

The stability of the axisymmetric equilibria throughout the pedestal region is observed. This is do to the weak or negative magnetic shear allowing for access to the secondary stability region of ballooning theory [35]. With the application of magnetic perturbations, the instability appears radially localized at the top of the edge pedestal, as seen in figure 4a matching the experimentally measured radial location of the instability. Figure $4 \mathrm{~b}$ demonstrates how the radial wavenumber, $\theta_{k}$, is localized for the 3D system, with the typical ballooning behavior of 3D MHD equilibria described in Ref. [20].

The toroidal localization of the mode under 3D magnetic perturbation is also observed in Figure 4 The ballooning growth rates are observed to have a significant dependence on the field line label, $\alpha$. The instability is seen to be $N=2$ periodic, matching the behavior of the 3D kink response. As the instability is enhanced at only a few toroidal angles, we can assert that the changes in 3D shaping have modified the local ballooning stability along specific bands of field lines, as seen in the experiment [19].

Calculations of the plasma displacement at the flux surface of interest are used to identify the position of the unstable field lines. Figure 5 shows the normal 3D plasma displacement, $\xi_{n}$, on 
the unfolded axisymmetric flux surfaces in straight field line ballooning coordinates, as well as a mapping of the unstable field line for the given surface. The instability localizes to the same zero-crossing in $\xi_{n}$ as observed in the experiment. This is seen in the 2D plots for figure 5, which show the localization of the eigenvalues, as well as the time trace of the ECE diagnostic from the experiment and the results of a synthetic ECE diagnostic for the computational data. The experimental data shows clear ballooning behavior with the same localization of the instability as we see in the computational results, all at the same location where the growth rates are toroidally localized [18].

Analysis has been carried out for all 27 equilibria given in table 1 . While details vary, the results of the case shown in Figure 4 are representative of all of the equilibria. The ballooning instability is localized in both radius and field line label indicating the effect of the 3D field is to locally distort the equilibrium so that only a few field lines are destabilized.

\subsection{Growth rate dependence on pressure modifier}

The tuning parameter $\hat{p}$ is used to make small scale modifications to the pressure profile without the need to fully recalculate the equilibria. In this study, we utilize the $\hat{p}$ parameter to statically shift the pressure profile until axisymmetric equilibrium is marginally stable. The marginal stability point was chosen as the target since experimental equilibria were found to be stable under axisymmetric operation, so we presume this to be the upper limit on the pressure profiles. Additionally, running at the upper limit in the pressure profiles eased achieving proper convergence of the ballooning eigenvectors, especially in the lower $\beta$ cases. It is expected that lower pressures are also unstable with the same localized trends, but the limits in our numerical methods make them difficult to observe. Finally, there is uncertainty in the experimental pressure profiles, which are averages as the pressure profile modulates quickly in time and space.

It is worth addressing the dependence of the growth rates on the $\hat{p}$ parameter, as this is not a self-consistent modification to the equilibrium. Physically, $\hat{p}$ shifts the pedestal height up or down, without changing any of the equilibrium magnetics, allowing us to estimate the effects of small changes in the pressure and improve numerical convergence.

Figure 6 shows the ballooning growth rates for various values of $\hat{p}$ for the 33345 equilibrium with $\Delta \varphi_{U L}$ of $-93^{\circ}$. We see that as we increase the pressure factor, the width and height of unstable region increase fairly dramatically. For the largest pressure cases, the $n=0$ equilibrium is also unstable (horizontal dotted lines), while the smallest cases are well below marginal stability. This shows how small changes in the pressure modifier can have measurable impact on the ballooning localization, as well as the underlying axisymmetric equilibria. Regardless of these changes, the toroidal localization of the instability remains unchanged, with only the width of the unstable region increasing while the location of the maximum growth rate remains constant. This solidifies our assumption that small changes to the pressure do not drastically change the underlying physics mechanism leading to the toroidal localization of the instability.

\subsection{Growth rate dependence on differential phase of MP coils}

The magnitude of 3D ballooning localization is seen to depend directly on the magnitude of the 3D plasma displacement at the unstable flux surface. The 3D displacement amplitude is controlled by the differential phase of the perturbation coils, $\Delta \varphi_{U L}$. Figure 7 provides an overview of results 
for all 27 equilibria. Comparison of the maximum displacement on the flux surface of interest (Fig. $7 \mathrm{a}$ to the growth rate of the most unstable field line (Fig. $7 \mathrm{~b}$ ) for each equilibria shows a strong correlation between stability and perturbation amplitude.

The relationship between the local growth rates and the displacement is best observed through direct comparison. Plotting the maximum growth rates against the maximum local 3D displacement (Figure 8) shows a linear dependence of the growth rates on the 3D perturbation, as expected. However, we observe a minimum amount of 3D displacement (3 to $4 \mathrm{~mm}$ for these cases) is needed before the mode becomes unstable locally. This means the changes in the local 3D geometry need to be significant enough to modify the local stability properties of the plasma, the impact of which will be assessed in the following sections.

The growth rates for the highest- $\beta$ case (Shot 33345) are seen to be greater than those of the lowest $\beta$ case (33118). However, the intermediate- $\beta$ case (33569) shows a decrease in the ballooning growth rate beyond that expected from pressure gradient drive alone. This deviation can be attributed to the significantly decreased displacement when compared to the other two cases, further showing how closely the localization of the ballooning mode is tied to the 3D displacement amplitude and its associated changes to the geometry.

\subsection{Energy dependence of the instability}

Utilizing a $\delta \mathrm{W}$-like formulation allows one to quantify the competition between the stabilizing and destabilizing contributions to the ballooning instability. The infinite-n ballooning equation is a simple 1D Sturm-Liouville equation, written as [20]

$$
\frac{\partial}{\partial \theta}\left[P(\theta) \frac{\partial X}{\partial \theta}\right]+Q(\theta) X=-\lambda R(\theta) X
$$

where the coefficient functions are defined as

$$
P(\theta)=\frac{1}{\sqrt{g}} \frac{\left(1+\Lambda^{2}\right)}{|\nabla \psi|^{2}} \quad Q(\theta)=\beta^{\prime} \sqrt{g} \frac{\left(\kappa_{n}+\Lambda \kappa_{g}\right)}{|\nabla \psi|} \quad R(\theta)=\sqrt{g} \frac{\left(1+\Lambda^{2}\right)}{|\nabla \psi|^{2}} \quad \lambda=\omega^{2}
$$

The eigenvalue $\lambda$ is related to the shape of the eigenfunction $X$ through the equation

$$
\lambda=\frac{\int_{-\infty}^{\infty} P(\theta)\left(\frac{\partial X}{\partial \theta}\right)^{2} d \theta-\int_{-\infty}^{\infty} Q(\theta) X^{2} d \theta}{-\int_{-\infty}^{\infty} R(\theta) X^{2} d \theta} .
$$

This shows that the growth rates are a balance between the stabilizing line-bending energy $\left(\bar{W}_{L B}\right)$ and the destabilizing pressure-curvature potential $\left(\bar{W}_{P C}\right)$

$$
\begin{gathered}
\bar{W}_{L B}=\int_{-\infty}^{\infty} \delta W_{L B} d \theta=\int_{-\infty}^{\infty} P(\theta)\left(\frac{\partial X}{\partial \theta}\right)^{2} d \theta \\
\bar{W}_{P C}=\int_{-\infty}^{\infty} \delta W_{P C} d \theta=\int_{-\infty}^{\infty} Q(\theta) X^{2} d \theta
\end{gathered}
$$

Evaluation of the energy terms, seen in table 2, shows how the 3D localization changes the available free energy leading to destabilization. The stabilizing line-bending energy is decreased along unstable field-lines, and increased for the stable field lines. The same behavior is seen in 
the driving energy, though the changes are significantly smaller, leading to a small number of field-lines having a surplus of free energy and thus going unstable. Figure 9 elucidates this point, showing the relative changes in the energy densities along the field-line for the most stable and most unstable field-lines (relative to the axisymmetic equilibrium). While there is variation in the pressure curvature drive (Fig. 9c), the dramatic changes are associated with the stabilizing field line bending contribution (Fig. $9 \mathrm{~b}$, which is substantially decreased along the field line.

The observed behavior in the mode energy provides us valuable insight into the mechanism behind the 3D destabilization of the ballooning instabilities. Figure $9 \mathrm{a}$ shows the integrated local magnetic shear along the stable and unstable field lines. Both the drive and the stabilizing terms have a dependence on the integrated magnetic local shear $(\Lambda)$, with the stabilizing term having a stronger $\left(\Lambda^{2}\right)$ dependence, as seen in equation 4 . We see that there is a large variation in the integrated local magnetic shear between the different field lines, clearly impacting the energy density terms seen in figure 9 . This demonstrates that the local magnetic shear is playing a key role in the localization of the ballooning instability.

\begin{tabular}{c|c|c|c} 
Case & $\bar{W}_{L B}$ & $\bar{W}_{P}$ & $\bar{W}_{T O T}$ \\
\hline Axi & 2.22 & -2.02 & 0.20 \\
Stable & 3.31 & -2.42 & 0.89 \\
Unstable & 1.70 & -1.71 & -0.01
\end{tabular}

Table 2: Energy balance

\subsection{Analysis of local 3D geometry on stability}

The changes in the energy terms can be attributed to three terms, the normal curvature (Fig. 10a), the geodesic curvature (Fig. 10b), and the local magnetic shear (Fig. 10c), where the local magnetic shear and curvature are defined as

$$
s_{l}=\hat{b} \times \hat{n} \cdot \nabla \times(\hat{b} \times \hat{n}),
$$

and

$$
\kappa=\hat{b} \cdot \nabla \hat{b}=\kappa_{n} \hat{n}+\kappa_{g} \hat{b} \times \hat{n}
$$

respectively [36].

Figures 10 (d-f) show the local 3D toroidal variation of the geometric quantities, calculated by taking the difference between each point and the toroidal mean, normalized by the difference in the maximum and minimum of the quantity in the poloidal direction. We see that the 3D shaping of the plasma boundary has the largest impact on the local magnetic shear on the low field side of the device, while the curvature terms see only small variation, mostly away from the low field side midplane. As we saw in figure 5 , the instability is localized at $\alpha \approx 90^{\circ}, 270^{\circ}$. Figure $10 f$ shows the local magnetic shear decreasing significantly on these field lines near $\theta \approx 30^{\circ}$, while a significant increase in the local magnetic shear is observed on the $\alpha \approx 0^{\circ}, 180^{\circ}$ field lines, which are seen to have increased stability properties. Figure 10d shows changes in the normal curvature, but they are localized more to the top of the device. Figure 10e shows changes in the geodesic curvature on the low field side, but they are significantly smaller than the changes observed in the local magnetic shear. 
This toroidal variation in the local magnetic shear is the driving the change in the destabilizing energy. Additionally, the stabilizing energy is entirely dependent on the square of the integrated local magnetic shear, explaining why the toroidal variation in the stabilizing term is more dramatic.

Having identified the changing local magnetic shear as the dominant mechanism in the localized destabilization of the mode, we look to analyze the physical quantities that contribute to these changes to better understand the underlying physics mechanic at work. To do this, we follow the work of [36], which breaks down the shear into two contributing factors as

$$
s_{l}=\mu_{0} \frac{\mathbf{J} \cdot \mathbf{B}}{B^{2}}-2 \tau_{n}
$$

where $\tau_{n}$ is the normal torsion, a purely geometric term, given by

$$
\tau_{n}=-\hat{n} \cdot(\hat{b} \cdot \nabla)(\hat{b} \times \hat{n}) .
$$

and $\mathbf{J} \cdot \mathbf{B} / B^{2}$ is the local current density along the field line.

Examination of these terms (figure 11) shows that the dominate component to the local magnetic shear on the low field side is the normal torsion, while the contribution from the parallel current is smaller by comparison. This leads to the poloidal structure of the local magnetic shear to be determined predominantly by the normal torsion of the magnetic field.

Figure 11 also illustrates the toroidal variation of the components to the magnetic shear, where the local 3D variation the two shear terms are plotted. Here we see that the toroidal variation of both the parallel current and normal torsion are significant. The parallel current provides a moderate contribution to the shear, with a moderate decrease on the low-field side at the unstable field lines. However, this term is not aligned with the field lines, so its affect is reduced near the midplane. This is likely cause by the parallel current aligning to the field lines at the nearby dominant rational surface, which have a different shear than the most unstable flux surface shown. The normal torsion sees a large local increase on the unstable field lines near $\theta \approx 30^{\circ}$, which translates to the large decrease of the shear at that location.

While both components of the shear have toroidal variation, the contribution from the normal torsion is the more impactful on the localization of the instability. This is significant, as the normal torsion is dependent only on the local 3D magnetic geometry. This shows that the 3D shaping of the plasma itself is responsible for the changing stability properties along select field lines. This also helps explain why the instability is only observed at one of the zero-crossings of the 3D displacement: the direction of motion of the plasma boundary determines whether the normal torsion is increasing or decreasing locally, relative to the underlying local magnetic topology (seen in figure 11). In the stable case, the change cause the field lines to twist tighter, increasing the stored line-bending energy. In the unstable case, the field lines unwind until there is a small region where they are locally viewed as non-bending, allowing the ballooning instability to occur. The amount of change require for the instability to arise is a matter of how close to the stability boundary the plasma is prior to application of the magnetic perturbations.

\section{Discussion}

Toroidal localization of MHD instabilities has been observed in ASDEX-Upgrade experiments [18]. Application of infinite-n ballooning theory to 3D VMEC equilibria based on these experiments has 
predicted the existence and basic behavior of these ballooning instabilities. Experimentally, the instability localized to field lines where the edge plasma displacement is zero, but only in when the pedestal is moving inward towards the plasma core (when rotating the MPs in the positive toroidal direction). The second zero in the displacement (when the pedestal is moving outward with the same MP rotation) is observably stable, despite the same pressure gradient being present in both cases. This results is substantiated by ideal MHD analysis, which is found sufficient for studying the basic behavior of the localized instability.

A key feature to the 3D ballooning instability is its dependence on the strength of the applied magnetic perturbations for both the experimental and numerical observations. Most notable is the requirement of a minimum amount of 3D displacement to observe the localized instability. This behavior is seen in other aspects of these low collisionality 3D perturbation experiments, namely the ELM mitigation [37, 6]. These phenomena tend to scale linearly with the magnetic perturbation strength after the initial onset occurs [37]. It has been seen that ELM-mitigation is at its peak for $\Delta \varphi_{U L} \approx-90^{\circ}$ [19], as is the ballooning growth rate. These correlations raise new questions on the relationship, if any, between the localization of the ballooning instabilities and the ELM mitigation observed in these experiments.

Focusing on the ballooning instability, we use a $\delta W$-like approach to gain insight into the mechanism of the instability. It was seen that the localization of the instability is the results of the line-bending energy decreasing until it is no longer sufficiently balancing the pressure-curvature drive when the 3D magnetic perturbation is increased. This can be used to explain the threshold behavior mentioned above. Until a sufficient amount of 3D displacement is present, the stabilizing term remains large enough to balance the drive. Beyond this point, the difference between the drive and stabilizing terms grows linearly with the magnitude of the 3D displacement, leading to the linear growth of the localized instability.

Analysis of the local magnetic geometry provides further insight into the underlying physical mechanism behind the localization of the ballooning instability. It is observed that the curvature terms have negligible 3D variation, while the integrated magnetic shear, governed by the local magnetic shear, has strong variation with changing field lines, namely the effect of the shear is significantly reduced on field lines that are ballooning unstable. This results is expected, as it has previously been shown that 3D magnetic perturbations can significantly impact local geometry [22], which can subsequently impact edge stability and transport [12]. However, our result deviates from the above in that we consider non-resonant magnetic perturbations in which the stable kink response is optimized, allowing for different components of the local geometry to govern edge stability. Specifically, previous results focused on changes in the local magnetic shear driven by Pfirsch-Schlüter currents at dominant rational surfaces [12]. The increased kink response studied here leads to the normal torsion dominating the local 3D variation of the magnetic shear throughout the pedestal region, even in the absences of dominate rational surfaces. It is worth noting that VMEC equilibria do not properly handle localized sheet currents at rational surfaces, however, sufficient radial resolution has been shown to lead to well converged values for key local geometry terms [38, 28], which was accounted for in this work.

Three-dimensional equilibria modifying the ballooning stability along field lines is not unique to magnetically perturbed tokamak equilibria. In stellarator equilibria, incommensurate helicities present in key geometric quantities break the symmetry of the local magnetic geometry and produce localized ballooning instabilities that can significantly alter the ballooning stability boundaries 
[39, 30], as well as impact the underlying turbulence and micro-stability [40]. The axisymmetric equilibria studied here are stable to ballooning modes due to the weak shear in the pedestal providing access to the secondary stability region [35]. However, the 3D magnetic perturbations lead to the large kink response at the top of the pedestal, which in turn causes symmetry breaking changes to the local magnetic geometry, locally modifying the stability boundary along the lines of Ref. [30].

There are still a number of open questions with regards to the toroidal localization of ballooning modes in the Tokamak edge region. The local magnetic shear having strong 3D dependence has potential to affect the micro-turbulence and associated plasma transport, as discussed in Ref. [12]. The transport in the plasma edge and structure of the ballooning instability in question needs to be quantified before a link between the localize ballooning and ELM mitigation is made.

This localized ballooning behavior has been observed in a number of shots on ASDEX Upgrade [23]. However, it has been noticeably absent from DIII-D discharges with similar parameters [28]. Key differences between these cases are the amplitude of the kink response ( $3 \mathrm{~mm}$ on DIII-D versus $12 \mathrm{~mm}$ on AUG, despite similar size and MP) and the upper triangularity of the discharge, both of which could effect the 3D local geometry. Additionally, the DIII-D cases were operating in an ELM-suppressed regime, while the others were in mitigated regimes. More recent AUG ELM-suppressed discharges did not see the localized ballooning [23, 41]. As there is an open question to the relationship of the localized MHD instability and the ELM mitigation, it is possible that fully suppressed discharges do not exhibit localized ballooning behavior.

There is a large amount of literature that extends beyond the infinite-n ballooning theory in both axisymmetric tokamaks [42] and stellarator systems [20, 43, 44] to approximate the values of the global mode numbers for these systems, and provide further insight into the behavior of the instability beyond what is done in this paper. Additionally, finite Larmor radius effects have been shown to strongly effect the stability properties of highly localized structures [45]. The infinite-n ballooning analysis can be extended to include these effects, as well as to incorporate general extended MHD and two-fluid physics [45]. Alternatively, extended MHD codes such a the NIMROD code [46] have been used to study ballooning behavior with the inclusion of these effects [47]. The infinite-n ballooning analysis simply acts as a first step towards understanding the localized ballooning instability observed in ASDEX Upgrade, as well as a motivating factor to future studies with more complete models.

This research is supported in part by the U.S. Department of Energy (DOE) Office of Science, Office of Fusion Energy Sciences under grant no. DE-FG02-86ER53218. 


\section{References}

[1] Y. Liang et al. 2007, Phys. Rev. Lett., 98:265004.

[2] T. E. Evans et al. 2006, Nature Physics, 2:419.

[3] T. E. Evans et al. 2004, Phys. Rev. Lett., 92:235003.

[4] R. Nazikian et al. 2015, Phys. Rev. Lett., 114:105002.

[5] G. R. McKee et al. 2013, Nuclear Fusion, 53(11):113011.

[6] A. Kirk et al. 2015, Nuclear Fusion, 55(4):043011.

[7] W Suttrop et al. 2017, Plasma Physics and Controlled Fusion, 59(1):014049.

[8] W. Suttrop et al. 2018, Nuclear Fusion, 58(9):096031.

[9] C. Paz-Soldan et al. 2015, Phys. Rev. Lett., 114:105001.

[10] Yueqiang Liu, A. Kirk, Y. Gribov, M.P. Gryaznevich, T.C. Hender, and E. Nardon. 2011, Nuclear Fusion, 51(8):083002.

[11] M. Willensdorfer et al. 2017, Phys. Rev. Lett., 119:085002.

[12] T M Bird and C C Hegna. 2013, Nuclear Fusion, 53(1):013004.

[13] I.T. Chapman. 2013, Physics of Plasma, 20(5).

[14] C. C. Hegna. 2014, Physics of Plasmas, 21(7):072502.

[15] I.T. Chapman et al. 2014, Nuclear Fusion, 54(8):083007.

[16] R. A. Moyer et al. 2012, Nuclear Fusion, 52(12):123019.

[17] M. J. Lanctot et al. 2011, Physics of Plasmas, 18(5):056121.

[18] M Willensdorfer et al. 2016, Plasma Physics and Controlled Fusion, 58(11):114004.

[19] M. Willensdorfer et al. 2017, Nuclear Fusion, 57(11):116047.

[20] R. L. Dewar and A. H. Glasser. 1983, The Physics of Fluids, 26(10):3038-3052.

[21] C. C. Hegna and N. Nakajima. 1998, Physics of Plasmas, 5(5):1336-1344.

[22] T. M. Bird and C. C. Hegna. 2014, Physics of Plasmas, 21(10):100702.

[23] Matthias Willensdorfer et al. 2018, Plasma Physics and Controlled Fusion. accepted.

[24] P J McCarthy. 2012, Phys. Plasma, 6(1):3554.

[25] P J McCarthy and ASDEX Upgrade Team. 2012, Plasma Physics and Controlled Fusion, 54(1):015010.

[26] S. P. Hirshman and J. C. Whitson. 1983, The Physics of Fluids, 26(12):3553-3568.

[27] E. Strumberger, S. Günter, and C. Tichmann. 2014, Nuclear Fusion, 54(6):064019.

[28] R.S. Wilcox et al. 2017, Nuclear Fusion, 57(11):116003. 
[29] S. R. Hudson and C. C. Hegna. 2003, Physics of Plasmas, 10(12):4716-4727.

[30] C. C. Hegna and S. R. Hudson. 2002, Physics of Plasmas, 9(5):2014-2019.

[31] S. R. Hudson and C. C. Hegna. 2004, Physics of Plasmas, 11(9):L53-L56.

[32] S.R. Hudson, C.C. Hegna, and N. Nakajima. 2005, Nuclear Fusion, 45(4):271.

[33] R. Sanchez, S.P. Hirschman, J.C. Whitson, and A.S. Ware. 2000, Journal of Computational Physics, 161(2):576-588.

[34] S.P. Hirshman, W.I. van RIJ, and P. Merkel. 1986, Computer Physics Communications, 43(1):143 155.

[35] J.M. Greene and M.S. Chance. 1981, Nuclear Fusion, 21(4):453.

[36] C. C. Hegna. 2000, Physics of Plasmas, 7(10):3921-3928.

[37] A. Kirk et al. 2013, Nuclear Fusion, 53(4):043007.

[38] S. A. Lazerson and the DIII-D Team. 2015, Nuclear Fusion, 55(2):023009.

[39] P. Cuthbert and R. L. Dewar. 2000, Physics of Plasmas, 7(6):2302-2305.

[40] B. J. Faber, M. J. Pueschel, P. W. Terry, C. C. Hegna, and J. E. Roman. 2018, Journal of Plasma Physics, 84(5):905840503.

[41] Leuthold et al. 2018, 45th EPS Conference on Plasma Phys.

[42] R.L. Dewar, J. Manickam, R.C. Grimm, and M.S. Chance. 1981, Nuclear Fusion, 21(4):493.

[43] D. Correa-Restrepo. 1978, Zeitschrift für Naturforschung A, 33(7):789-791.

[44] W.A. Cooper, D.B. Singleton, and R.L. Dewar. 1996, Physics of Plasmas, 3(1):275-280.

[45] R. J. Hastie, Peter J. Catto, and J. J. Ramos. 2000, Physics of Plasmas, 7(11):4561-4566.

[46] C. R. Sovinec, T. A. Gianakon, E. D. Held, S. E. Kruger, and D. D. Schnack. 2003, Physics of Plasmas, 10(5):1727-1732.

[47] B. J. Burke, S. E. Kruger, C. C. Hegna, P. Zhu, P. B. Snyder, C. R. Sovinec, and E. C. Howell. 2010, Physics of Plasmas, 17(3):032103. 


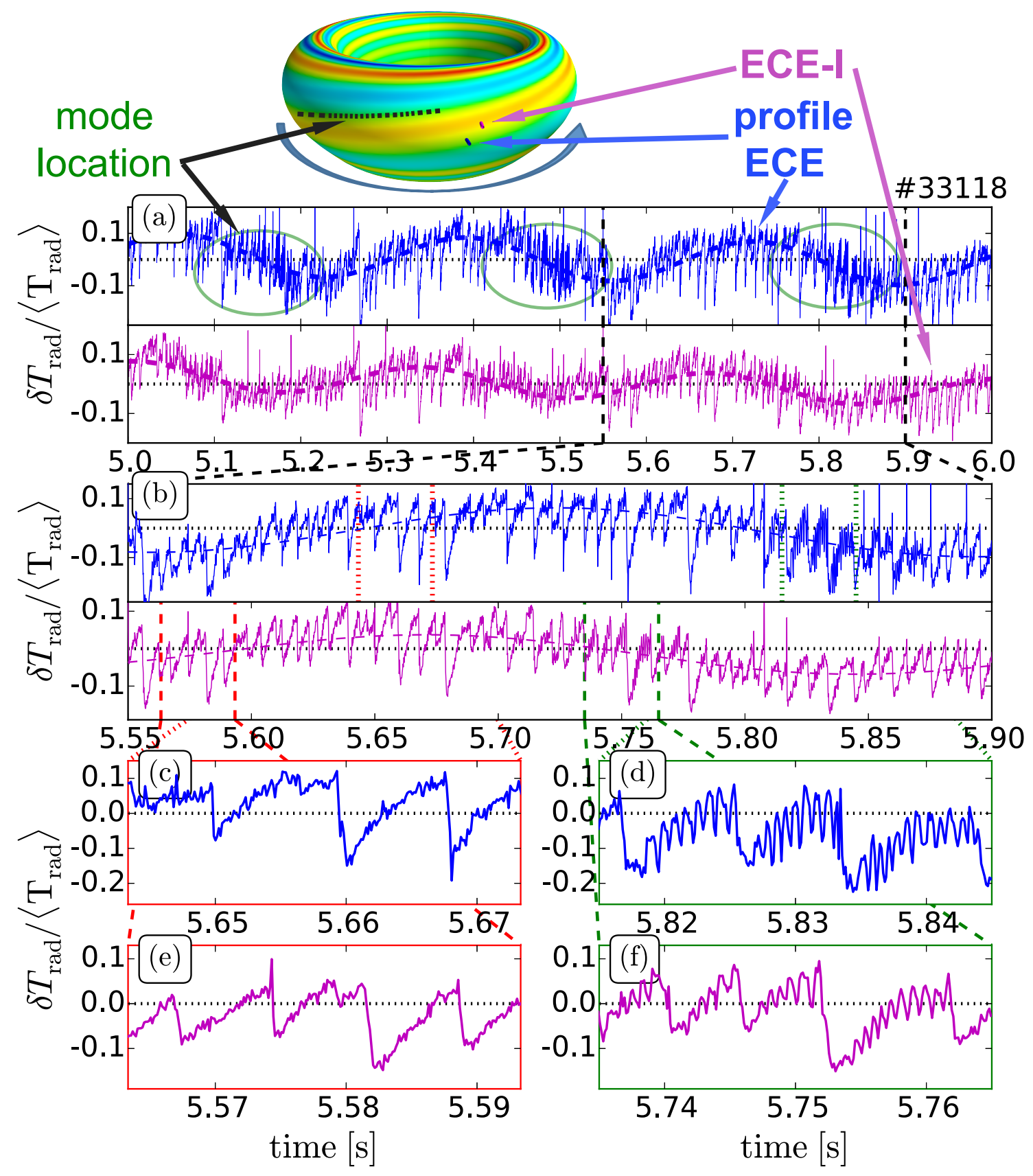

Figure 1: Time traces from profile ECE (blue) and ECE-I (purple) channels. Horizontal dotted lines indicate the zero line. Measuring principle and line-of-sight positions are indicated at the top. (a) A $3 \mathrm{~Hz}$ modulation due to the rotating $\xi_{r} ;(b)$ a MHD mode with $f \approx 1 \mathrm{kHz}$ is seen when one specific $\xi_{r} \approx 0$ [indicated by green arrows in (a)] passes the diagnostics. The mode appears in between ELM crashes and at only one $\xi_{r} \approx 0$ $(d),(f)$, whereas at the other times not, e.g., $(c),(e)$. Please note the different time ranges between $(c)-(f)$ illustrated by shaded areas in (b). Taken from Ref. [11]. 


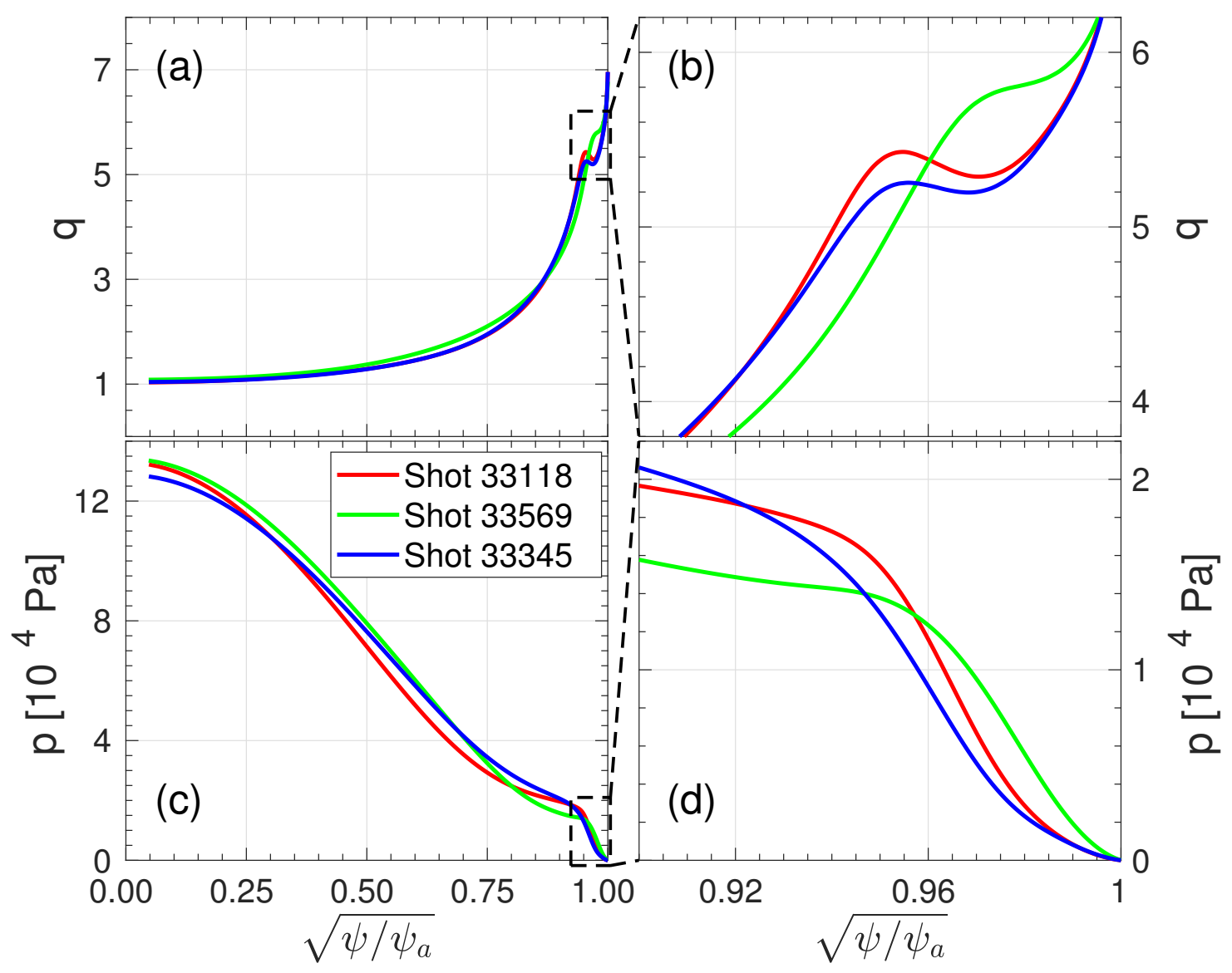

Figure 2: ASDEX Upgrade derived 2D H-mode profiles for $(a),(b) q$ and $(c),(d)$ the pressure from CLISTE. $(b)$ and $(d)$ show the profiles in the edge pedestal region where the ballooning modes are located. 


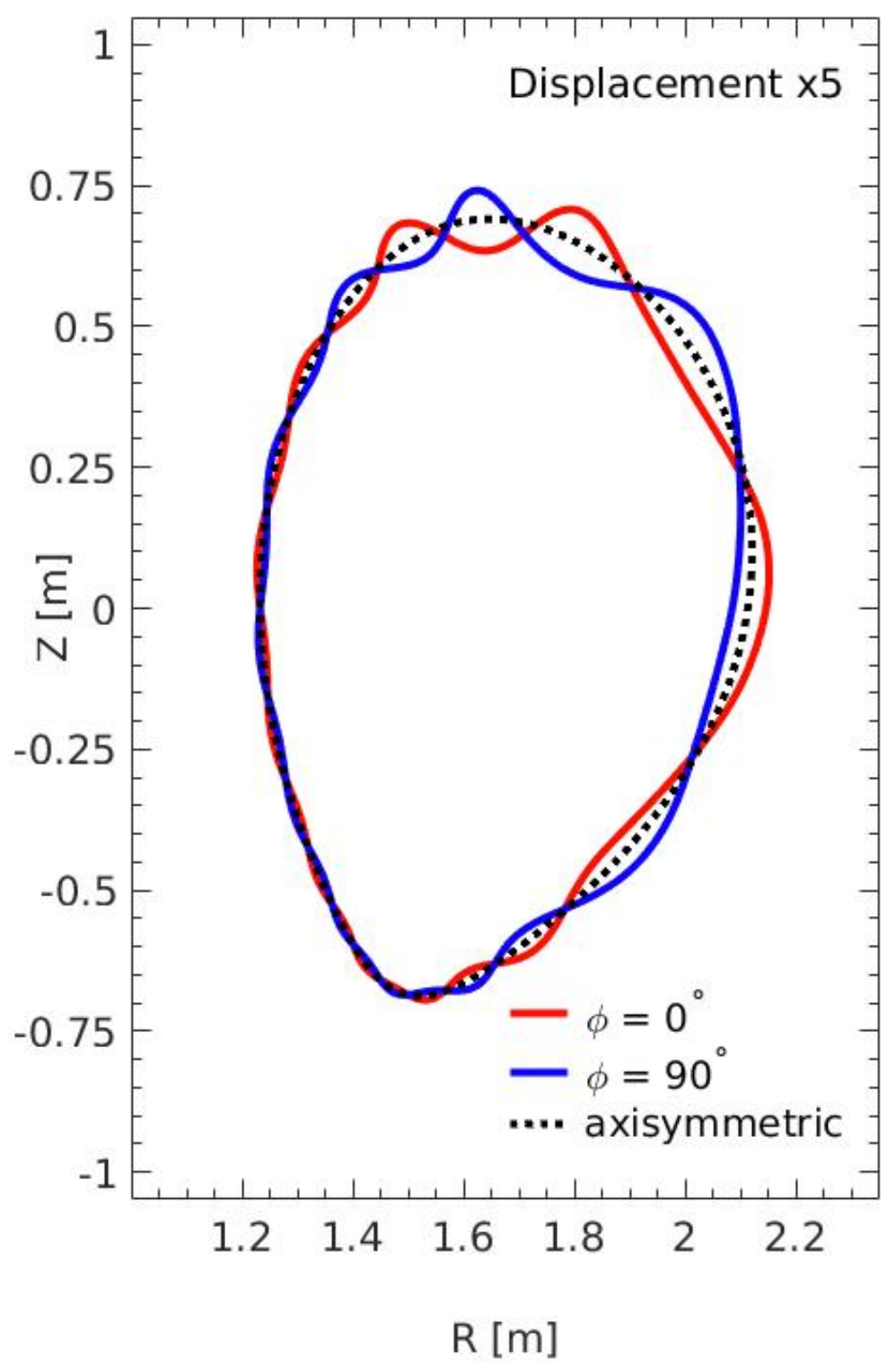

Figure 3: Edge displacement of the $\psi=0.95$ flux surface for Shot 33345 equilibrium with $\Delta \varphi_{U L}=-93^{\circ}$. The displacement is largest on the low-field side of the discharge. 


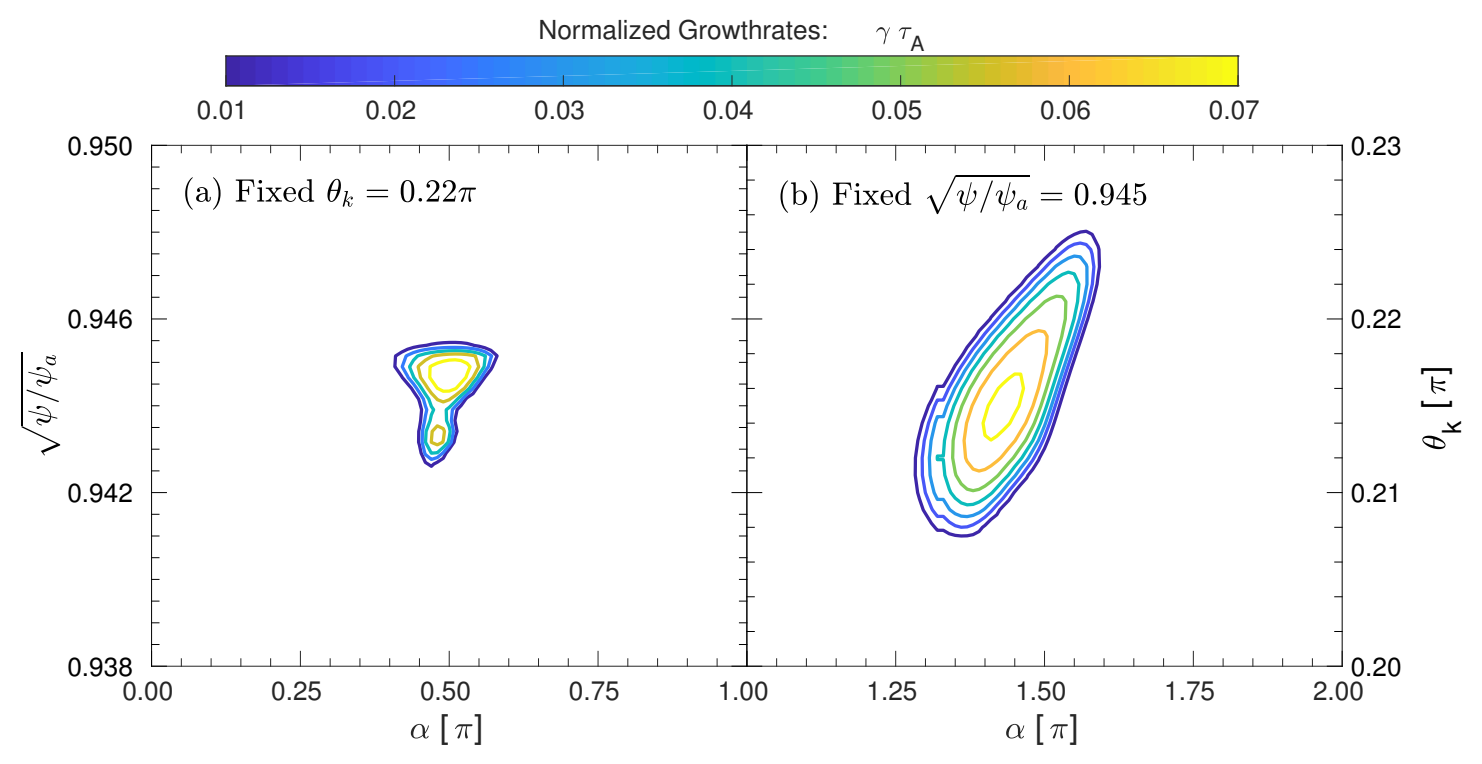

Figure 4: Ideal ballooning growth rates for Shot 33345 equilibrium with $\Delta \varphi_{U L}=-93^{\circ}$. (a) Variation of $\psi$ and $\alpha$ with fixed $\theta_{k}=0.22 \pi$ and $(b)$ variation of $\theta_{k}$ and $\alpha$ with fixed $\sqrt{\psi / \psi_{a}}=0.945$. 

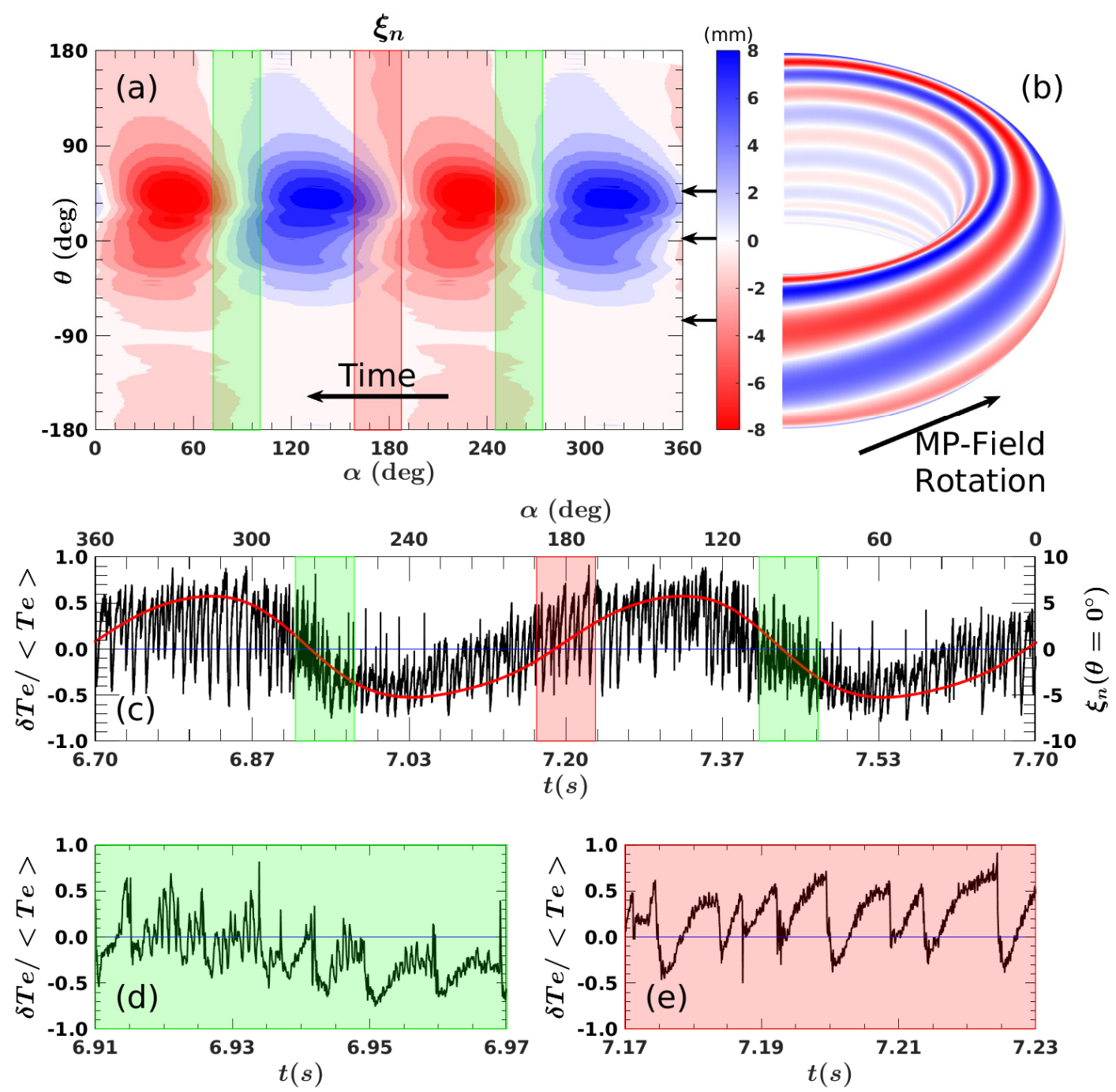

Figure 5: Normal plasma displacement on (a) the unfolded and (b) toroidal flux surface at $\sqrt{\psi / \psi_{a}}=0.945$. (c) Displacement (red) overlayed on the ECE data for shot 33345. Green and red boxes denote the most unstable and most stable regions (respectively), all located at zero crossings of the displacement based on computational results with (d) and (e) showing close up of the ECE data for these regions. Figure (a) utilizes straight field line coordinates, with the $X$-point $\left(\theta=-75.4^{\circ}\right)$, outboard mid-plane $\left(R_{\max }, \theta=2.45^{\circ}\right)$, and plasma top $\left(Z_{\max }, \theta=46.6^{\circ}\right)$ identified by the arrows to the right of the top figure. 

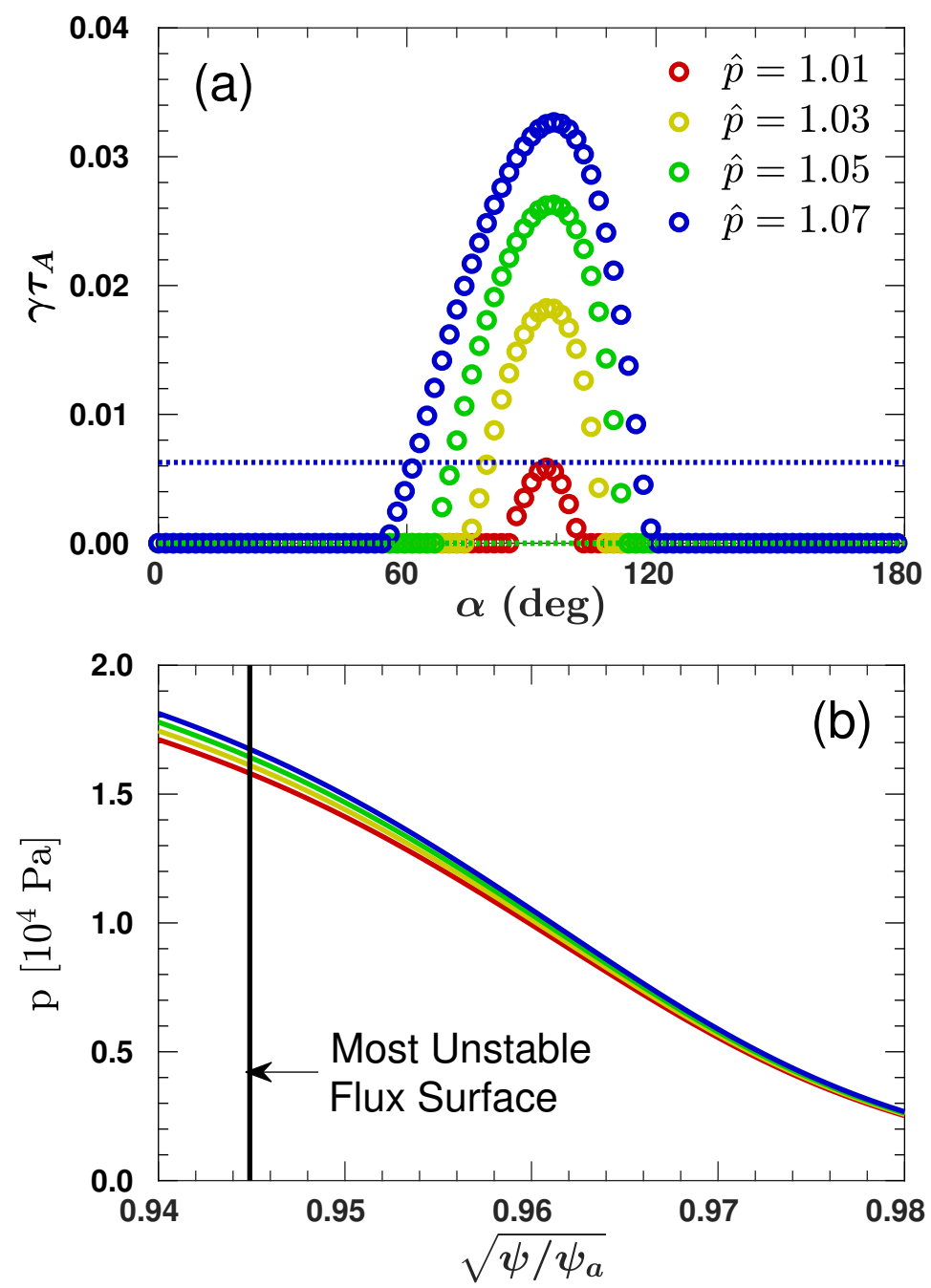

Figure 6: (a) Dependence of the ballooning growth rate on the pressure modifier, $\hat{p}$, as well as (b) the modified pedestal pressure profiles versus normalized poloidal flux. The horizontal dotted line in (a) designates the growth rate of the axisymmetric equilibria for the $\hat{p}=1.07$ case, while all other cases are stable for the $2 D$ equilibria. 


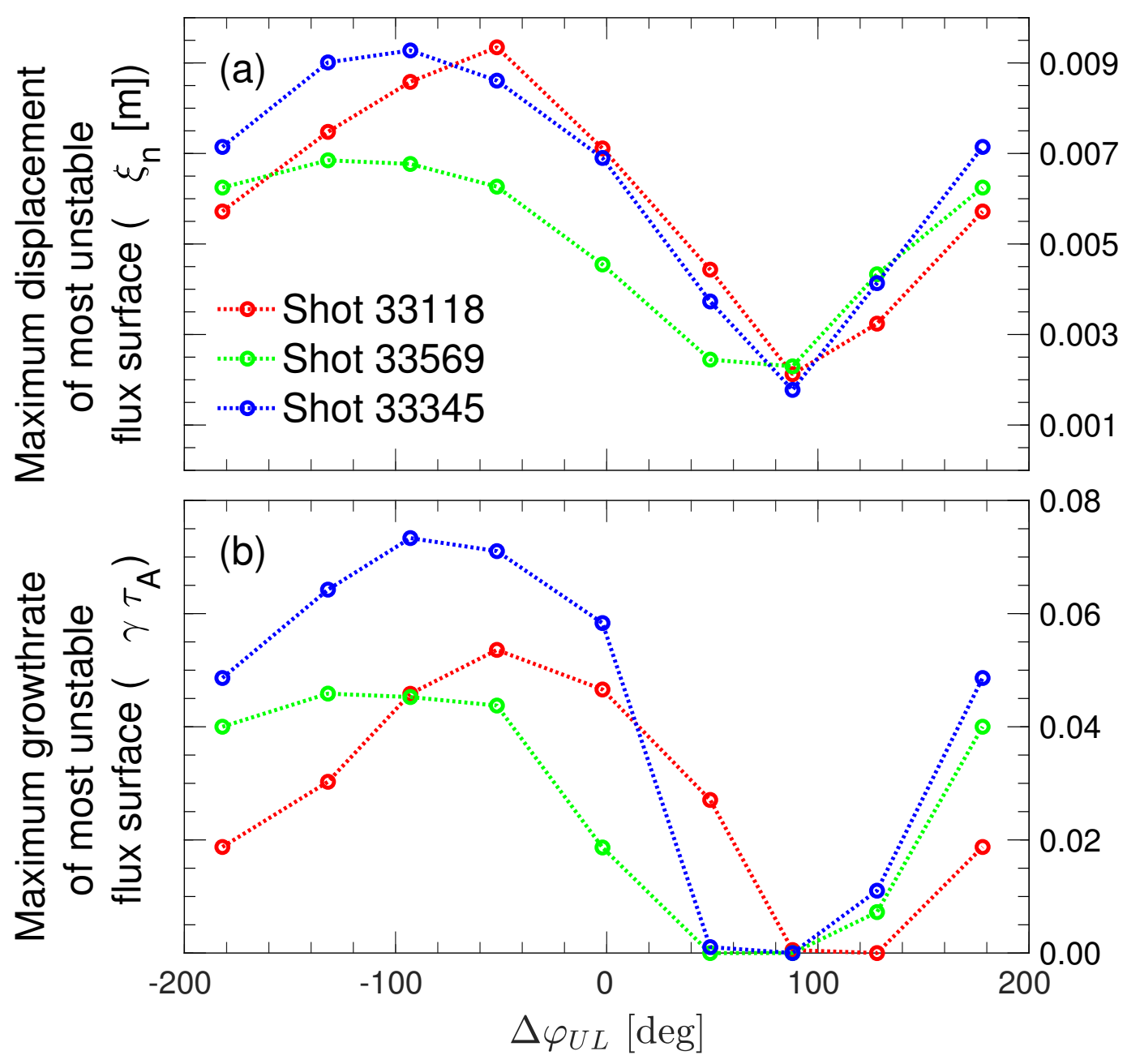

Figure 7: Dependence of (a) the maximum 3D displacement at the unstable flux surface and $(b)$ the maximum ballooning growth rate on the alignment of the magnetic perturbation coils. 


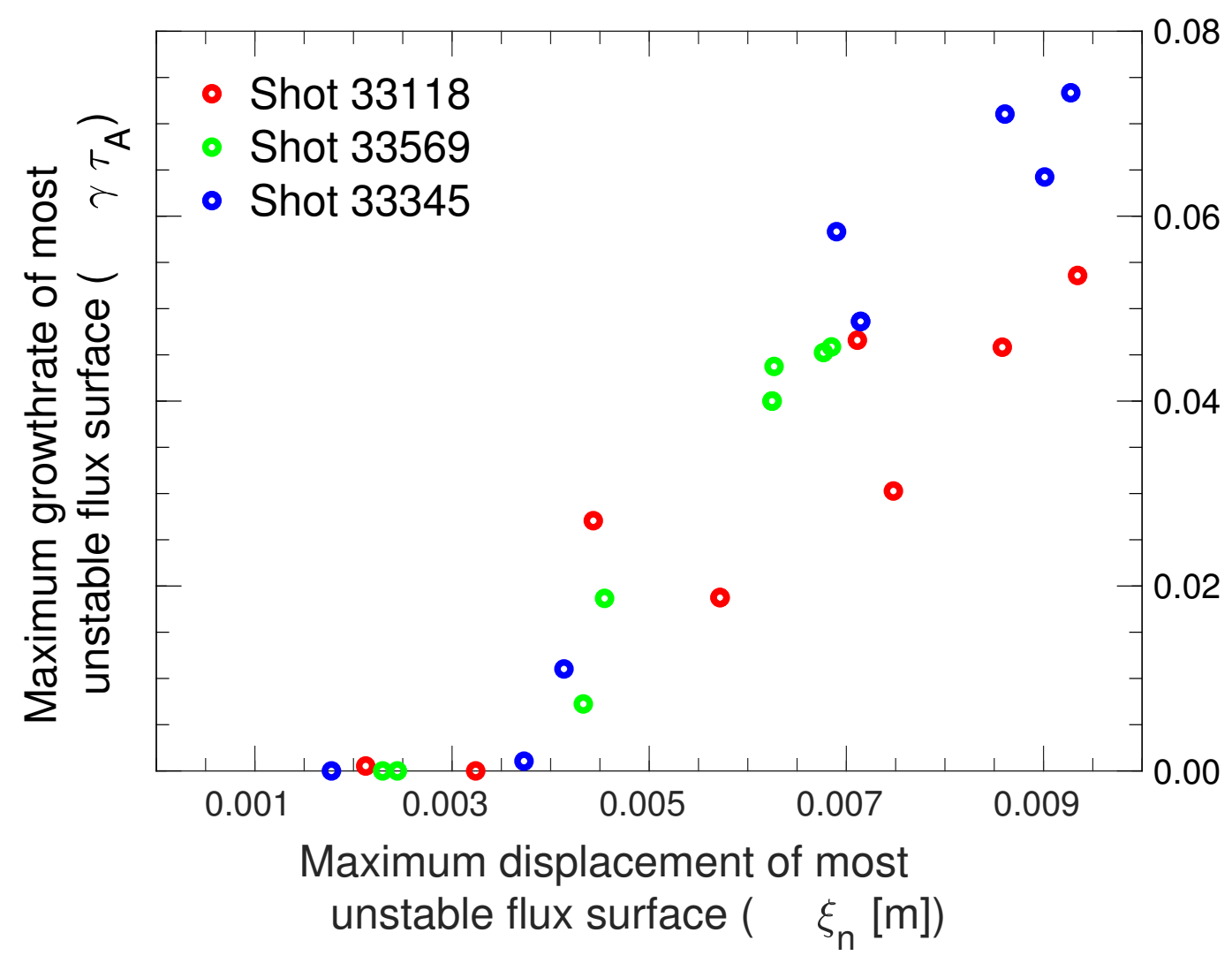

Figure 8: Dependence of the maximum ballooning growth rate on the maximum of the 3D displacement of the unstable flux surface. 

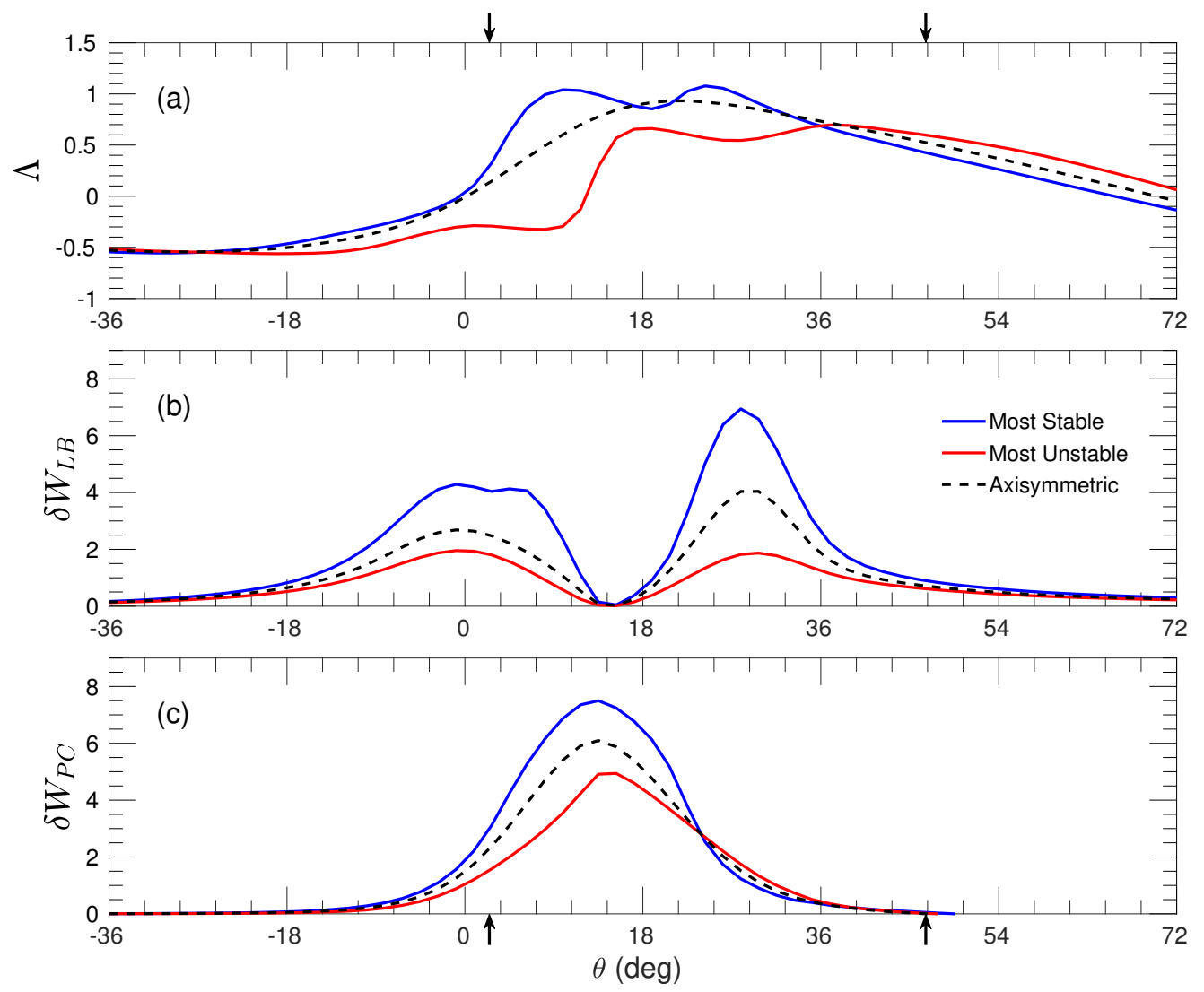

Figure 9: (a) Integrated local magnetic shear, and the (b) stabilizing line bending and (c) destabilizing pressurecurvature drive components of the energy density for equilibrium shot 33345 with $\Delta \varphi_{U L}=-93^{\circ}$ at $\sqrt{\psi / \psi_{a}}=0.945$ flux surface. The arrows show the outboard mid-plane $\left(R_{\max }, \theta=2.45^{\circ}\right)$ and plasma top $\left(Z_{\max }, \theta=46.6^{\circ}\right)$. 

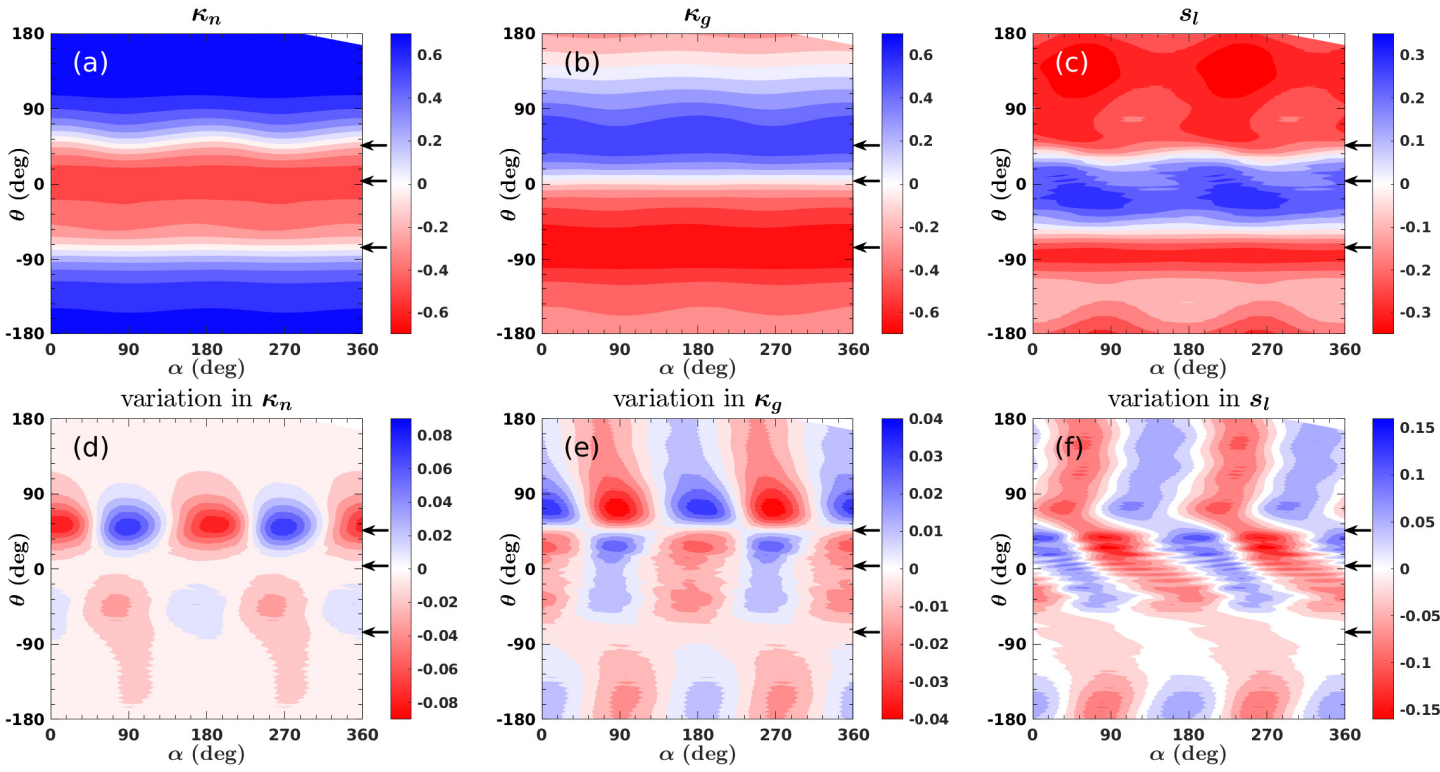

Figure 10: (a) Normal curvature, (b) geodesic curvature, and (c) local magnetic shear. (d-f) show the ratio of the toroidal to poloidal variation of the corresponding components in $(a-c)$. All quantities are for shot 33345 with $\Delta \varphi_{U L}=-93^{\circ}$ plotted with the straight field line coordinates at $\sqrt{\psi / \psi_{a}}=0.945$. The arrows to the right of the figures show the $X$-point $\left(\theta=-75.4^{\circ}\right)$, outboard mid-plane $\left(R_{\max }, \theta=2.45^{\circ}\right)$, and plasma top $\left(Z_{\text {max }}, \theta=46.6^{\circ}\right)$. 

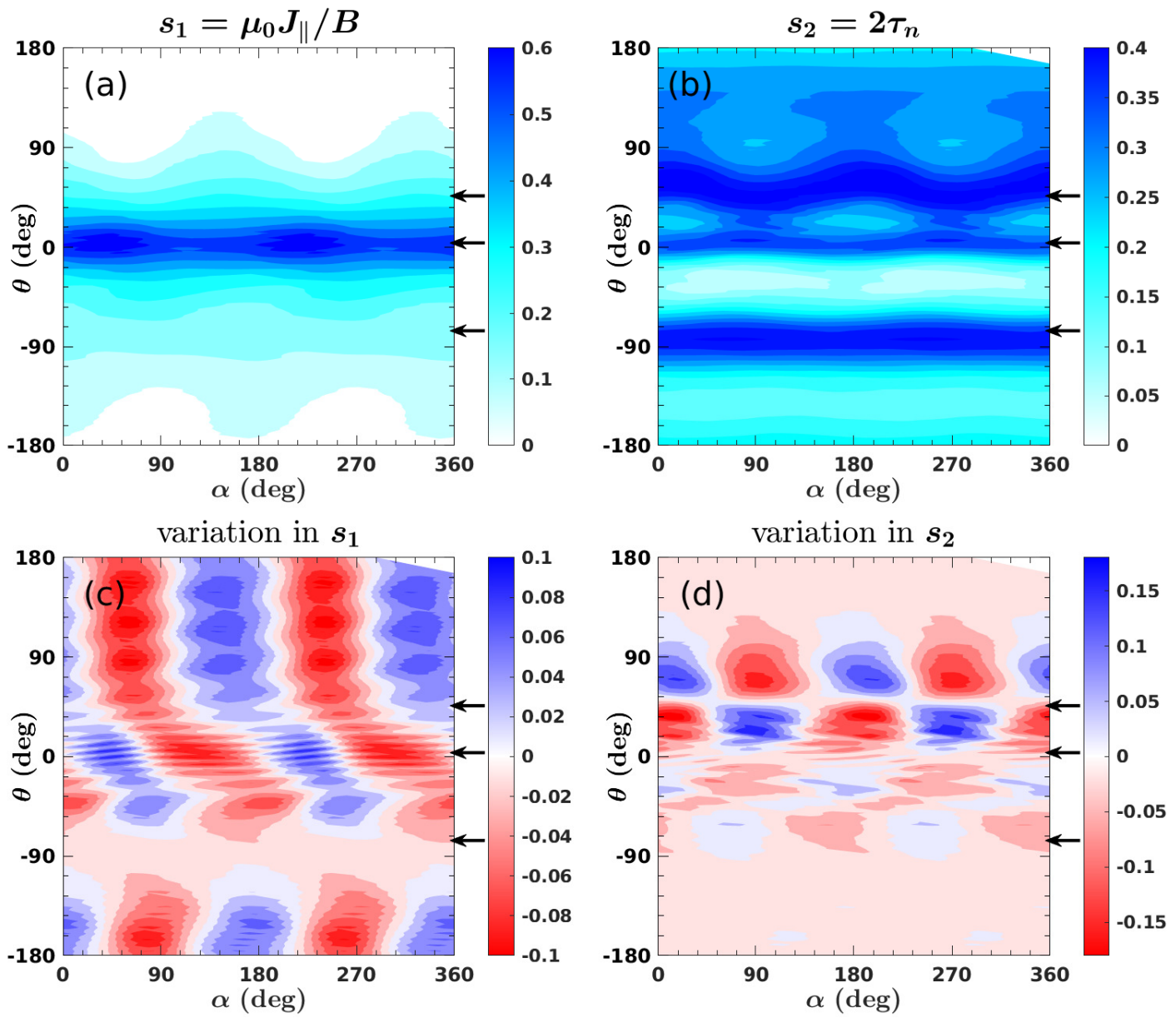

Figure 11: (a) Parallel current and (b) normal torsion contributions to the local magnetic shear $\left(s_{l}=s_{1}-s_{2}\right)$. (c),(d) show the ratio of the toroidal to poloidal variation of the corresponding components in $(a),(b)$. All quantities are for shot 33345 with $\Delta \varphi_{U L}=-93^{\circ}$ plotted with the straight field line coordinates at $\sqrt{\psi / \psi_{a}}=0.945$. The arrows to the right of the figures show the X-point $\left(\theta=-75.4^{\circ}\right)$, outboard mid-plane $\left(R_{\max }, \theta=2.45^{\circ}\right)$, and plasma top $\left(Z_{\max }, \theta=46.6^{\circ}\right)$. 